although in the ordinary subject they are in the other direction under the same conditions, while the eye movements are presumably the same in all cases, a new line of interpretation is opened up.

Boswell's experiments were on the apparent changes of shape which certain spots of light undergo when moved rapidly across the retina with the eyes fixed. By means of a pendulum device spots of various forms and not exceeding $I^{\circ} 1 O^{\prime}$ of visual angle were moved at various rates over an area including practically only the fovea. Under these conditions the upper and lower portions of the figure appear displaced backwards, so that, for instance, a vertical bar appears as a crescent. Boswell believes that this is due to irradiation, which strengthens the central region of the lighted area, causing it to come to consciousness before the peripheral regions, and hence spatially in advance of them. To establish this explanation, Boswell tried darkening the middle regions of certain spots, and was able by proper darkening to prevent the backward displacement of the upper and lower portions, or to produce a forward displacement, although the amount of darkening necessary is hardly perceptible with the figure at rest. Spots of light longer vertically than the ones mentioned above gave a straight middle region with curved ends, since the irradiation along the middle region was nearly uniform.

By an ingenious arrangement of cross wires, Boswell measured the apparent curvature of a vertical band at different speeds and with different intensities and colors of light. He found that with increasing intensities the displacement at first increased and then decreased. Green gave the greatest maximum of displacement, red and blue the least, yellow being about midway between.

In the speculative part of the paper Boswell expresses the belief that the irradiation takes place in retinal layers more inward than the rods and cones, approves W. McDougall's neurological theory as a provisional scheme, and conjectures a central origin for color blindness.

\title{
Feeling.
}

The Expression of Feelings: F. M. URBAN.

The Mutual Influence of Feelings: JoHN A. H. KHITH.

The Combination of Feelings: C. H. Johnston.

The Esthetics of Repeated Space Forms: Eleanor Harris RowLand.

The Feeling-Value of Unmusical Tone-intervals: L. E. Emerson.

Urban states at the beginning of his paper that the material for it was obtained by an experimental investigation carried on in the Har- 
vard laboratory, but he gives no information as to the nature of the experiment. The paper is in substance a discussion of the nature and possible causes of the dicrotic elevation of the arterial pulse, with an excellent account of the several theories and of the study of the pulse from Marey's experiments to the present day. Urban decides that the cause of the dicrotic elevation is that which Landois assigned to the pre-dicrotic and post-dicrotic elevations, namely, arterial elasticity.

Keith presents with the least possible comment the results of a long series of experiments with two subjects on the hedonic ranking of various colors, tone combinations, and surfaces actively or passively touched. Twenty-seven tone combinations and fourteen of each of the other groups were separately given their values in the conventional scale of seven degrees of pleasantness-unpleasantness, and then the members of each group were combined in turn with members of the other groups and assigned values under those conditions. The general averages indicate that combining colors and tones lowers the agreeableness of both, while combining colors and passive touch or tones and active touch raises the agreeableness of both in both cases. For the other combinations the results are not concordant. No details of the effects of individual members of one group on the various members of the others are given.

Johnston's article is in many respects the complement to Keith's. The experimental results presented are merely a mass of introspection from twelve subjects, either on viewing Perry Pictures, or experiencing combinations of odors, tones, noises, touches, and space forms, a member from each of two of these groups being used in each combination. The deliverances of the subjects in attempting to tell the way things felt to them are an interesting study in elevated metaphor. Apparently neither subjects nor author spared words. The main problem seems to have been to find if two feeling tones could be present at once and if the general conditions of complex feeling tones agree with the Münsterburg Action-Theory, on both of which questions the author concludes affirmatively.

The first part of Miss Rowland's paper reports the introspection of seven subjects on viewing regularly repeated groupings of lines. The lines were white strings hung across a black background, shifted through a great variety of groupings. Miss Rowland thinks the introspection shows that the properties of repeated space-forms are analogous to those of auditory rhythm as the latter are set forth by MacDougall. The second part of the paper is devoted to a study of approved architecture, attempting to show that it embodies the same 
principles as are brought out in the first part. Whether in either part the author makes out her case is not to be answered offhand. The material is presented with little regard for clearness, conciseness or unity, so that the reader can hardly escape getting lost in the diffuse miscellany of details.

Mr. Emerson experimented with what he designates by the unfortunate term 'amusical' melodies; $i$. e., series of three notes on two pitches including an interval varying from $460: 45^{6}$ to $460: 516$, and - from $3^{8} 4: 43^{6}$ to $3^{8} 4: 5^{16}$, by steps of four. The subjects estimated the values of the 'melodies' in the seven-degree hedonic scale, and afterwards estimated independently the harmony of the two pitches. Later, some experiments were made with from three to six notes on three pitches differing by 4,8 and 12 vibrations, absolute pitch not stated. The results show that the intervals used do give decided feeling tones in most cases; that the preferences for melody do not correspond to those for harmony; and that the preferred melodic intervals within the range indicated are (according to Emerson), from four to eight vibrations less than the 'half or full tone of the musical scale,' and likewise fail to correspond with the musical intervals from the second to the fourth.

Emerson apparently takes account of major tones and semitones only, in computing musical intervals, and hence does not notice that the preferences are approximately for the minor tone and semitone. The paper is gotten up carelessly, to say the least, and the reader is left in doubt about many points. In plates showing melody-curves one may identify them with some trouble after he has decided whether the intervals marked are diatonic, chromatic, or tempered; but the significance of two sets of harmony-curves in one plate is entirely obscure, as is the reference to 'Tables I. and II.,' no tables being discernible.

\section{Association, Apperception, Attention.}

Certainty and Attention: Frances H. Rousmaniere.

Inhibition and Reinforcement: Lours A. TURLEY.

The Interference of Optical Stimuli: (Miss) H. KLEINKNecht. Subjective and Objective Simultaneity: Thomas H. Haxnes.

The Estimation of Number: C. T. BurNeTt.

Time-Estimation in its Relation to Sex, Age, and Physiological

Rhythms: R. M. YeRKES and F. M. URBAN.

Association under the Infuence of Different Ideas: BrRD $\mathrm{T}$. Baldwin.

Dissociation: C. H. ToLL. 